\title{
SUBMISSION TYPE
}

Poster

TITLE

How to Use Item Desirability Ratings in Constructing Forced-Choice Tests

SHORTENED TITLE

Item Desirability Ratings and Forced-Choice Tests

\begin{abstract}
The forced-choice response format as faking prevention method relies on matching items comprising each forced-choice block in terms of desirability for the job. We argue that utilizing mean desirability ratings for item matching may yield inaccurate similarity values and result in inferior matches. We propose a distance-based alternative that may yield optimal matches.
\end{abstract}

\section{PRESS PARAGRAPH}

The forced-choice response format has been proposed as a method for preventing applicant faking on self-report non-cognitive measures. This potential benefit of the format depends on how closely the items comprising each forced-choice block are matched in terms of desirability for the job. Current desirability matching procedures rely on differences in items' mean desirability ratings to quantify similarity in items' desirability. We argue that relying on means, while ignoring individual differences in desirability ratings, may yield inaccurate similarity values and result in inferior item matches. As an alternative, we propose a distance-based measure that considers differences in desirability ratings at the individual level and may thus yield accurate similarity values and optimal matches. We support our arguments on a set of desirability ratings obtained with an explicit instruction to rate desirability of items.

WORD COUNT

2791

APA CITATION

Pavlov, G., Shi, D., \& Maydeu-Olivares, A. (2020). How to Use Item Desirability Ratings in Constructing Forced-Choice Tests [Poster]. Society for Industrial and Organizational Psychology Annual Conference, Austin, TX, United States. 


\section{How to Use Item Desirability Ratings for Constructing Forced-Choice Tests}

The forced-choice response format is a common method for preventing applicant faking on non-cognitive measures in employee selection settings. Unlike the Likert (aka rating) response format, which allows applicants willing to fake to agree with indicator items that are favorable for the job and disagree with the unfavorable ones, the forced-choice format, by design, does not allow applicants to (dis)agree with all the (un)favorable items comprising the same forced-choice block. However, in order to qualify as a potentially effective faking prevention device, the format also needs to incorporate an additional design feature. Specifically, items comprising each forced-choice block should be matched in terms of their desirability for the job. It is hoped that together with this additional feature, aimed at completely depriving respondents from making response choices based on items' desirability, the format might "force" applicants to respond based on something else, hopefully on true levels of the measured constructs (Dilchert \& Ones, 2011; Saltz, Reece, \& Ager, 1962).

Matching items in terms of desirability has been a common practice in forced-choice test construction, especially for measures intended for employee selection. Matching procedures currently in use routinely involve first obtaining items' desirability ratings on a "desirability sample". Then, mean desirability is computed for each item under consideration. Finally, each forced-choice block is formed by combining items with "sufficiently" similar mean desirability values, often in conjunction with other considerations such as item discrimination, location, and/or content (e.g., Chernyshenko et al., 2009; Christiansen, Burns, \& Montgomery, 2005; Converse et al., 2010; Lee, Joo, \& Lee, 2019; Lee, Joo, Stark, \& Chernyshenko, 2019; Lee, Lee, 
\& Stark, 2018; Stark, Chernyshenko, \& Drasgow, 2005; Usami, Sakamoto, Naito, \& Abe, 2016; Vasilopoulos, Cucina, Dyomina, Morewitz, \& Reilly, 2006).

Despite recent proliferation of measures in the forced-choice response format intended for various high-stakes assessments and personnel selection in particular (e.g., Drasgow et al., 2012; Naemi, Seybert, Robbins, \& Kyllonen, 2014; Watrin, Geiger, Spengler, \& Wilhelm, 2019), evaluating and/or improving desirability matching procedures has received little research attention. Given the critical role of desirability matching in creating fake-resistant forced-choice measures, the purpose of the current study was to address this outstanding need in the forcedchoice literature.

\section{Item Desirability Matching}

Desirability matching procedures rely on empirically obtained desirability information. Commonly, respondents in a desirability sample are either explicitly asked to rate desirability of each item under consideration (e.g., Chernyshenko et al., 2009; Christiansen et al., 2005; Usami et al., 2016), or asked to respond to items under a fake-good instruction set with a strong manipulation to fake (e.g., Drasgow et al., 2012; Stark et al., 2005). In either approach, the obtained ratings are averaged across respondents to arrive at one desirability value for each item under consideration.

The next stage of item desirability matching routinely involves computing absolute differences between items' mean desirability ratings. The absolute difference in mean desirability ratings is the key metric in the process of desirability matching. It quantifies the similarity between items in terms of desirability in units of the rating scale used (Kilmann \& Thomas, 1977), with smaller absolute mean differences indicating items perceived as more similar. We refer to this metric here as the mean difference measure. Finally, the mean difference 
measure is used for selecting item-pairs, commonly by setting a specific mean difference value that is considered the maximal acceptable dissimilarity between items in terms of desirability, and then considering only item-pairs with the mean difference below the chosen cutoff (e.g, see Chernyshenko et al., 2009; Drasgow et al., 2012; Vasilopoulos et al., 2006).

\section{The Mean Difference Measure}

Formally put, the mean difference measure is given by:

$$
\left|\bar{d}_{k}-\bar{d}_{j}\right|
$$

where $\bar{d}_{k}$ and $\bar{d}_{j}$ are mean desirability ratings for items $k$ and $j$, and $\left|\bar{d}_{k}-\bar{d}_{j}\right|$ is the absolute difference in mean desirability ratings between the two items. The result of Equation in (1) is commonly interpreted as the extent of similarity between items in terms of desirability, expressed in units of the rating scale used, with a value of 0 indicating items with identical desirability.

The mean difference measure given in Equation (1) can be equivalently expressed as follows:

$$
\frac{\left|\sum_{i=1}^{N}\left(d_{i, k}-d_{i, j}\right)\right|}{N},
$$

where $d_{i, k}-d_{i, j}$ are differences between individual desirability ratings of items $k$ and $j$, and $N$ is the size of the desirability sample.

It is critical to observe in Equation in (2) that the mean difference measure relies on the assumption that the differences in individual desirability ratings between items $k$ and $j$, i.e., $d_{i, k}-d_{i, j}$, will not be of the opposite sign. If this assumption is violated, the differences of the opposite sign will be canceling each other out. In that case, the mean difference measure would 
no longer be able to adequately capture the differentiation between items at the individual level and might thus yield inaccurate similarity values. For instance, if respondent $\mathrm{X}$ rates desirability of item A with a 3 and desirability of item B with a 5, and respondent $Y$ rates item A with a 5 and item B with a 3, the result of Equation in (2) will be 0, indicating identical desirability of items A and B. However, both respondents in the example, in fact, do not perceive the two items as equally desirable.

\section{A Distance-based Desirability Measure}

As discussed previously, due to a strong underlying assumption, the mean difference measure may result in inaccurate similarity values. In order to eliminate this possibility, we propose an similarity alternative metric:

$$
\operatorname{Block}_{(k, j)}=\sum_{i=1}^{N}\left|d_{i, k}-d_{i, j}\right|,
$$

where Block $(\mathrm{k}, \mathrm{j})$ is the Block distance between items $k$ and $j$. Instead of computing the sum of differences (see Equation (2)), Block distance computes the sum of absolute differences between individual desirability ratings of items $k$ and $j$, and by relaxing the assumption inherent in the mean difference measure more adequately evaluates similarity between items in terms of desirability.

To facilitate the interpretation of the similarity values obtained with the Block distance, we may propose the averaged Block distance:

$$
\text { Block }_{\operatorname{avg}(k, j)}=\frac{\text { Block }_{(k, j)}}{N} .
$$

Block $_{\text {avg }}$ can be interpreted as the average difference between individual desirability ratings of items $k$ and $j$, in units of the rating scale used. 


\section{An Empirical Illustration}

In the previous section, we demonstrated mathematically that the mean difference measure may lead to misleading values of similarity between items in terms of desirability. As an alternative, we proposed a distance-based measure, i.e., Block $\mathrm{avg}_{\mathrm{g}}$ that overcomes potential shortcoming inherent in the mean desirability measure. In the following empirical example, we attempted to ascertain if the undesirable feature of the mean difference measure may represent a concern in practical applications.

\section{Method}

We used desirability data from the study by Pavlov, Maydeu-Olivares, and Fairchild (2019). The study measured Big Five personality dimensions with the Forced Choice Five Factor Markers questionnaire (Brown \& Maydeu-Olivares, 2011) and its rating version. Both versions consist of sixty items with twelve indicators measuring each Big Five personality dimension, eight of which are positively keyed and four reversely keyed. In the study, one hundred and eighty undergraduate psychology students from a large public university in Spain were asked to explicitly rate desirability of each item against a specified job target on a 5-point scale ranging from "very undesirable" (-2) to "very desirable" (2).

\section{Results}

In Table 1, we present summary statistics for all sixty items. Items in the table are ordered with respect to the mean desirability rating, from highest to lowest. The mean desirability values ranged from 1.7500 to -1.7333 . Because of the coding the response alternatives, items with mean desirability values above zero were on average rated as desirable, and items with the mean values below zero as undesirable for the target job. It can be observed in the table that the positively 
keyed items tended to have higher mean desirability values than the negatively keyed items; the opposite was true for Neuroticism items.

Correspondence of desirability indices. We computed the mean desirability measure (termed "|diff|" throughout the analysis) with Equation (2), and the averaged Block distance $\left(\right.$ Block $_{\text {avg }}$ ) with Equation (4). The total number of item-pairs was $\left(\begin{array}{c}60 \\ 2\end{array}\right)=1770$. All computations were performed in R (R Development Core Team, 2015).

The obtained |diff| values ranged from 0 to 3.4833 , and Block $\mathrm{avg}_{\text {values ranged from }}$ .2778 to 3.4833. We then computed differences between Block $k_{\text {avg }}$ and |diff| (Block avg $_{\text {- }}$ diff|) for all 1770 item-pairs. These differences ranged from 0 to .9333 , with an average difference of .1953. In terms of the rating scale used, this finding revealed that |diff| was returning larger values than Block $\mathrm{avg}_{\mathrm{ag}}$, or specifically, was overestimating similarity by approximately .2 units on average, and in some instances as much as almost 1 full unit of the 5-point rating scale.

To examine this further, we created a bivariate scatterplot of $\mid$ diff $\mid$ against the differences between Block avg and |diff|. This plot is presented in Figure 1. The dotted line in the figure

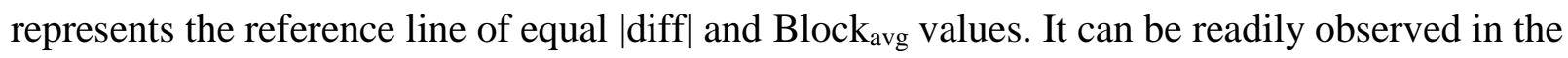
figure that item-pairs with large |diff| values are falling either on the reference line or relatively close to it. This finding indicated that the larger mean difference values were closely corresponding with Block $\mathrm{avg}_{\text {. }}$ Conversely, the figure shows that the discrepancy of the two indices was pronounced the most for item-pairs with the smallest mean difference values. It was particularly concerning that for some of the "ideal" item-pairs $(|\operatorname{diff}| \approx 0)$ the difference between Block $_{\text {avg }}$ and |diff| was as large as 1 unit of the 5-point rating scale, thus suggesting potential substantial desirability differentiation at the individual level. 
Item-pair selection decisions based on the two indices. In practical applications, test developers most often specify an absolute mean difference cutoff value, which represents a maximal acceptable dissimilarity between items' desirability in units of the ratings scale, and then consider only item-pairs with the mean difference value below the chosen cutoff (e.g, Chernyshenko et al., 2009; Drasgow et al., 2012; Vasilopoulos et al., 2006). We compared this item-pair selection strategy with selection based on Block avg. Based on the cutoff values used in previous studies, we chose cutoffs that select only item-pairs with the mean difference value below $.07, .35$, and .70 units of the 5-point rating scale. We refer to the chosen cutoffs as stringent, moderate, and loose, respectively.

The item-pair selection accuracy was evaluated as follows. First, we selected all itempairs with |diff| values equal or below each of the three chosen cutoffs. The number of selected item-pairs under the $.07, .35$, and .70 cutoff was 108,525 , and 794 respectively. We provide summary results for the selected item-pairs in the upper part of Table 2 . We then selected the same number of item-pairs $(108,525$, and 794) with the lowest Block avg values. Summary results for these item-pairs are provided in the middle part of Table 2. Finally, for each cutoff, we computed the percentage of item-pairs selected using Block avg $_{\text {that }}$ were also selected with |diff|. The selection correspondence results are presented in the lower part of the table.

It is readily observed in Table 2 that the selection correspondence relying on the mean difference measure and Block $\mathrm{avg}_{\mathrm{a}}$ was a function of the stringency of the cutoff used. Specifically, under the stringent cutoff $(|\operatorname{diff}|=.07)$, the selection based on mean differences included only $39.81 \%$ of the 108 most similar item-pairs identified with Block $\mathrm{avg}_{\text {g }}$. Under the moderate cutoff $(|\operatorname{diff}|=.35)$, the selection correspondence substantially improved $(81.14 \%)$. Finally, when the 
loose cutoff was used $(|\operatorname{diff}|=.70)$, selection correspondence based on the two indices was the best $(96.73 \%)$.

Because item-pair selection results under the stringent cutoff were most concerning, and because stringent matching is commonly desired the most in practical applications, we evaluated the "quality" of the selected item-pairs obtained under this selection regime. The average |diff| value for the 108 item-pairs selected with the mean difference measure was .0351 (see Table 2). In terms of the rating scale used, this result indicated that on average, the difference in desirability between the selected item-pairs was approximately .04 units of the 5-point scale. From the practical standpoint, this mean difference could be considered negligible. However, the average Block $_{\text {avg }}$ for those same item-pairs was .5566. Therefore, item-pairs with absolute mean differences below .07 had, on average, an average difference in individual desirability ratings of approximately .56 units of the 5-point scale. In addition, as shown in Table 2, the maximum

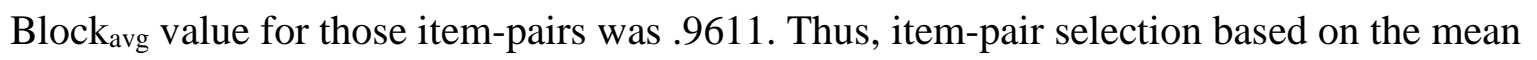
difference measure and the cutoff value of only .07 was allowing for item-pairs with an average difference in individual desirability ratings of up to approximately 1 full unit of the 5-point scale to be selected.

As shown in Table 2, when Block ${ }_{\mathrm{avg}}$ was used to select the same number (108) of itempairs, average Block $k_{a v g}$ values for the selected pairs was .4026. Thus, item-pairs selected with Block $_{\text {avg }}$ had smaller average differences in individual desirability ratings than item-pairs selected with the mean difference measure, on average by approximately .16 units of the 5-point scale (or approximately $30 \%$ lower). Moreover, the maximum Blockavg value was .4778 . Thus, item-pair selection based on Blockavg was allowing for item-pairs with the average difference in 
individual desirability ratings of roughly up to .5 units of the 5-point scale to be selected, half of what was allowed with selection relying on the mean difference measure.

\section{Discussion}

Administering non-cognitive self-report measures in the forced-choice response format might be particularly beneficial in high-stakes settings, such as employee selection, where characteristics of the situation may drive respondents to engage in faking, that is, opt for response options that convey favorable self-descriptions (Paulhus, 1984). If forced-choice blocks consist of items that are similar in terms of desirability, faking might be reduced or even altogether eliminated. Item desirability matching is routinely performed on empirically derived desirability ratings, by computing the mean desirability rating for each item under consideration. Absolute differences in mean desirability ratings are then used as a measure of similarity between items in terms of desirability to drive selection of the most similar item-combinations. In the present study, we have discussed the appropriateness of this similarity measure, referred to as the mean difference measure, and have proposed a distance-based alternative.

As shown, the mean difference measure relies on the assumption that differences in individual desirability ratings between items will not be of the opposite sign. If this assumption is violated, the measure might yield inaccurate similarity values, because differences in individual desirability ratings of the opposite sign will be canceling each other out. On a set of desirability ratings, obtained with an explicit instruction to rate item desirability, we have demonstrated that the mean difference measure may fail in practical applications. Specifically, accuracy of the mean difference measure might be compromised the most for item-pairs with the most similar mean desirability ratings. This finding raises a serious concern, because it challenges the 
common belief that combining items with very similar mean desirability ratings will ensure very close item matching (e.g., Feldman \& Corah, 1960).

We also evaluated the most commonly employed item-pair selection strategy, which selects pairs with the absolute mean differences below a predetermined mean difference cutoff. While this item-pair selection strategy performed similarly to the distance measure when a relatively loose cutoff value was used, its performance showed discrepancies with the increased cutoff stringency. Hence, if a more stringent matching is desired, relying on the mean difference measure might be especially problematic. Specifically, not only that many of the closest itempairs may be left unselected, but also item-pairs of notably suboptimal similarity might be selected instead.

The effectiveness of the forced-choice format in preventing faking has received limited empirical support in research to date, with findings being rather mixed (for review see, e.g., Christiansen et al., 2005; Pavlov et al., 2019). Considering results presented here, we suspect that relying on the mean difference measure for item desirability matching in previous studies might have contributed substantially to the inconsistencies in research findings. In addition, considering issues inherent in the mean difference measure reported in the present study, i.e., its potential to overestimate similarity between items, we suspect that previous research, on aggregate, has somewhat underestimated the utility of the forced-choice format to counter desirability driven responding.

As an alternative to the mean difference measure, we have proposed a distance-based measure, that is, Blockavg. As demonstrated, Blockavg relaxes the assumption inherent in the mean difference measure and therefore represents a more reliable measure of similarity between items in terms of desirability. Our findings showed that relying on Block $k_{\text {avg }}$ may result in a superior 
item-pair selection, especially when a more stringent desirability matching is desired. In addition, Block $_{\text {avg }}$ can be computed with any available statistical software package, it relies on desirability information that can be obtained via traditional desirability collection designs, and finally, it can be utilized not only for development of new forced-choice measures, but also for improving the existing ones.

\section{References}

Brown, A., \& Maydeu-Olivares, A. (2011). Forced-choice Five Factor markers. https://doi.org/10.1037/t05430-000

Chernyshenko, O. S., Stark, S., Prewett, M. S., Gray, A. A., Stilson, F. R., \& Tuttle, M. D. (2009). Normative scoring of multidimensional pairwise preference personality scales using IRT: Empirical comparisons with other formats. Human Performance, 22(2), 105127. https://doi.org/10.1080/08959280902743303

Christiansen, N. D., Burns, G. N., \& Montgomery, G. E. (2005). Reconsidering forced-choice item formats for applicant personality assessment. Human Performance, 18(3), 267-307. http://dx.doi.org/10.1207/s15327043hup1803_4

Converse, P. D., Pathak, J., Quist, J., Merbedone, M., Gotlib, T., \& Kostic, E. (2010). Item Desirability Ratings in Forced-Choice Personality Measure Development: Implications for Reducing Score Inflation and Providing Trait-Level Information. Human Performance, 23(4), 323-342. https://doi.org/10.1080/08959285.2010.501047

Dilchert, S., \& Ones, D. S. (2011). Application of preventive strategies. In M. Ziegler, C. MacCann \& D.R. Roberts (Eds.), New perspectives on faking in personality assessment (pp. 177-200). New York: Oxford University Press. http://dx.doi.org/10.1093/acprof:oso/9780195387476.001.0001 
Drasgow, F., Stark, S., Chernyshenko, O. S., Nye, C. D., Hulin, C. L., \& White, L. A. (2012). Development of the tailored adaptive personality assessment system (TAPAS) to support army personnel selection and classification decisions. (Technical Report No. 1311). Arlington, VA: U.S. Army Research Institute for the Behavioral and Social Sciences. Retrieved from https://apps.dtic.mil/docs/citations/ADA564422

Feldman, M. J., \& Corah, N. L. (1960). Social desirability and the forced choice method. Journal of Consulting Psychology, 24(6), 480-482. http://dx.doi.org/10.1037/h0042687

Kilmann, R. H., \& Thomas, K. W. (1977). Developing a forced-choice measure of conflicthandling behavior: The" MODE" instrument. Educational and Psychological Measurement, 37(2), 309-325. https://doi.org/10.1177\%2F001316447703700204

Lee, P., Joo, S. H., \& Lee, S. (2019). Examining stability of personality profile solutions between Likert-type and multidimensional forced choice measure. Personality and Individual Differences, 142, 13-20. https://doi.org/10.1016/j.paid.2019.01.022

Lee, P., Joo, S.-H., Stark, S., \& Chernyshenko, O. S. (2019). GGUM-RANK Item and Person Parameter Estimation With Multidimensional Forced Choice Triplets. Applied Psychological Measurement, 43(3), 226-240. https://doi.org/10.1177/0146621618768294

Lee, P., Lee, S., \& Stark, S. (2018). Examining validity evidence for multidimensional forced choice measures with different scoring approaches. Personality and Individual Differences, 123, 229-235. https://doi.org/10.1016/j.paid.2017.11.031

Naemi, B., Seybert, J., Robbins, S., \& Kyllonen, P. (2014). Examining the WorkFORCETM Assessment for Job Fit and Core Capabilities of FACETS ${ }^{\mathrm{TM}}$. ETS Research Report Series, 2014(2), 1-43. https://doi.org/10.1002/ets2.12040 
Paulhus, D. L. (1984). Two-component models of socially desirable responding. Journal of Personality and Social Psychology, 46(3), 598. https://psycnet.apa.org/doi/10.1037/00223514.46.3.598

Pavlov, G., Maydeu-Olivares, A., \& Fairchild A. J. (2019). Effects of applicant faking on forcedchoice and Likert scores, Organizational Research Methods, 22(3), 710-739. http://dx.doi.org/10.1177/1094428117753683

R Development Core Team. (2015). R: A Language and Environment for Statistical Computing. R Foundation for Statistical Computing, Vienna, Austria.

Saltz, E., Reece, M., \& Ager, J. (1962). Studies of forced-choice methodology: Individual differences in social desirability. Educational and Psychological Measurement, 22(2), 365-370. https://doi.org/10.1177\%2F001316446202200209

Stark, S., Chernyshenko, O. S., \& Drasgow, F. (2005). An IRT approach to constructing and scoring pairwise preference items involving stimuli on different dimensions: The multiunidimensional pairwise-preference model. Applied Psychological Measurement, 29(3), 184-203. http://dx.doi.org/10.1177/0146621604273988

Usami, S., Sakamoto, A., Naito, J., \& Abe, Y. (2016). Developing Pairwise Preference-Based Personality Test and Experimental Investigation of Its Resistance to Faking Effect by Item Response Model. International Journal of Testing, 16(4), 288-309. http://dx.doi.org/10.1080/15305058.2016.1145123

Vasilopoulos, N. L., Cucina, J. M., Dyomina, N. V., Morewitz, C. L., \& Reilly, R. R. (2006). Forced-Choice personality tests: A measure of personality and cognitive ability? Human Performance, 19(3), 175-199. http://dx.doi.org/10.1207/s15327043hup1903_1 
Watrin, L., Geiger, M., Spengler, M., \& Wilhelm, O. (2019). Forced-Choice Versus Likert Responses on an Occupational Big Five Questionnaire. Journal of Individual Differences. Advance online publication. https://doi.org/10.1027/1614-0001/a000285 
Table 1

Summary Statistics for Sixty Five Factor Marker Items

\begin{tabular}{|c|c|c|c|c|c|c|c|c|c|c|c|}
\hline $\mathrm{I}$ & $\mathrm{T}$ & $\mathrm{M}$ & SD & Min & Max & I & $\mathrm{T}$ & $\mathrm{M}$ & SD & Min & Max \\
\hline 27 & $\mathrm{E}$ & 1.7500 & .4829 & 0 & 2 & 39 & A & .9500 & .8670 & -1 & 2 \\
\hline 42 & $\mathrm{O}$ & 1.6111 & .5430 & 0 & 2 & 19 & $\mathrm{O}$ & .8500 & .7435 & -1 & 2 \\
\hline 49 & A & 1.5944 & .5951 & -1 & 2 & 55 & $\mathrm{E}$ & .8444 & .7968 & -1 & 2 \\
\hline 23 & $\mathrm{O}$ & 1.5778 & .5589 & 0 & 2 & 34 & $\mathrm{~N}(\mathrm{r})$ & .8000 & .8869 & -2 & 2 \\
\hline 38 & $\mathrm{E}$ & 1.5611 & .5708 & -1 & 2 & 20 & $\mathrm{E}$ & .6444 & .7061 & -1 & 2 \\
\hline 11 & $\mathrm{C}$ & 1.5500 & .6097 & -1 & 2 & 41 & $\mathrm{E}$ & .5389 & .8006 & -1 & 2 \\
\hline 46 & $\mathrm{O}$ & 1.5500 & .6278 & -1 & 2 & 60 & $\mathrm{E}$ & .1889 & .6144 & -2 & 2 \\
\hline 36 & $\mathrm{O}$ & 1.4944 & .6558 & -1 & 2 & 37 & $\mathrm{O}(\mathrm{r})$ & -.4111 & .8442 & -2 & 2 \\
\hline 18 & $\mathrm{E}$ & 1.4889 & .5932 & -1 & 2 & 4 & $\mathrm{E}(\mathrm{r})$ & -.4500 & .8274 & -2 & 2 \\
\hline 3 & $\mathrm{O}$ & 1.4444 & .7996 & -2 & 2 & 21 & $\mathrm{C}(\mathrm{r})$ & -.8111 & .7824 & -2 & 1 \\
\hline 50 & $\mathrm{C}$ & 1.4389 & .6357 & 0 & 2 & 13 & $E(r)$ & -.8833 & .8474 & -2 & 2 \\
\hline 30 & $\mathrm{C}$ & 1.4056 & .6575 & -1 & 2 & 31 & $\mathrm{O}(\mathrm{r})$ & -.9722 & .8080 & -2 & 1 \\
\hline 43 & $\mathrm{C}$ & 1.3556 & .6569 & -1 & 2 & 9 & $\mathrm{O}(\mathrm{r})$ & -.9778 & .7907 & -2 & 2 \\
\hline 7 & $\mathrm{C}$ & 1.3500 & .6116 & -1 & 2 & 24 & $\mathrm{E}(\mathrm{r})$ & -1.0444 & .7386 & -2 & 1 \\
\hline 17 & $\mathrm{~N}$ & 1.3333 & .7247 & -1 & 2 & 5 & $\mathrm{O}(\mathrm{r})$ & -1.0667 & .7444 & -2 & 2 \\
\hline 57 & $\mathrm{C}$ & 1.3278 & .7683 & -2 & 2 & 53 & $\mathrm{~N}$ & -1.0778 & .6968 & -2 & 1 \\
\hline 56 & A & 1.3056 & .8194 & -1 & 2 & 47 & $\mathrm{C}(\mathrm{r})$ & -1.1500 & .6725 & -2 & 1 \\
\hline 15 & A & 1.2833 & .6624 & -1 & 2 & 40 & $\mathrm{~A}(\mathrm{r})$ & -1.1778 & 1.0257 & -2 & 2 \\
\hline 22 & $\mathrm{C}$ & 1.2833 & .6366 & -1 & 2 & 54 & $A(r)$ & -1.2722 & .8312 & -2 & 2 \\
\hline 33 & A & 1.2722 & .6754 & -1 & 2 & 8 & $A(r)$ & -1.2778 & .8784 & -2 & 2 \\
\hline 58 & $\mathrm{C}$ & 1.2667 & .6818 & -2 & 2 & 59 & $A(r)$ & -1.3556 & .8431 & -2 & 2 \\
\hline 2 & $\mathrm{E}$ & 1.2611 & .6961 & -1 & 2 & 32 & $\mathrm{~N}$ & -1.4111 & .6324 & -2 & 0 \\
\hline 44 & $\mathrm{O}$ & 1.2000 & .8078 & -2 & 2 & 51 & $\mathrm{~N}$ & -1.5056 & .6208 & -2 & 2 \\
\hline 1 & $\mathrm{~N}(\mathrm{r})$ & 1.1833 & .7584 & -1 & 2 & 52 & $\mathrm{C}(\mathrm{r})$ & -1.5500 & .5418 & -2 & 1 \\
\hline 10 & A & 1.1611 & .7025 & -1 & 2 & 25 & $\mathrm{C}(\mathrm{r})$ & -1.5611 & .7025 & -2 & 2 \\
\hline 29 & $N(r)$ & 1.1111 & .7760 & -2 & 2 & 14 & $\mathrm{~N}$ & -1.5778 & .6068 & -2 & 2 \\
\hline 16 & A & 1.1000 & .8975 & -1 & 2 & 45 & $\mathrm{~N}$ & -1.5833 & .6152 & -2 & 2 \\
\hline 12 & $\mathrm{O}$ & 1.0944 & .7818 & -2 & 2 & 28 & $E(r)$ & -1.6056 & .6208 & -2 & 2 \\
\hline 26 & $\mathrm{~N}(\mathrm{r})$ & 1.0722 & 1.0466 & -2 & 2 & 6 & $\mathrm{~N}$ & -1.6667 & .5285 & -2 & 0 \\
\hline 35 & A & 1.0111 & .7622 & 0 & 2 & 48 & $\mathrm{~N}$ & -1.7333 & .5243 & -2 & 1 \\
\hline
\end{tabular}

Note. $\mathrm{N}=180 ; \mathrm{I}=$ item; $\mathrm{T}=$ trait measured; $\mathrm{M}=$ mean; $\mathrm{SD}=$ standard deviation; $\mathrm{Min}=$ minimum; $\mathrm{Max}=$ maximum; $\mathrm{N}=$ Neuroticism $; \mathrm{E}=$ Extraversion $; \mathrm{O}=$ Openness to Experience; $\mathrm{A}=$ Agreeableness $; \mathrm{C}=$ Conscientiousness; $(\mathrm{r})=$ reversely keyed item. 
Table 2

Item-pair Selection Results Based on the Mean Difference Measure and Blockavg

\begin{tabular}{|c|c|c|c|c|c|c|c|c|c|c|c|c|}
\hline Method & \multicolumn{12}{|c|}{$|\operatorname{diff}|$} \\
\hline |diff| cutoff & \multicolumn{4}{|c|}{.07} & \multicolumn{4}{|c|}{.35} & \multicolumn{4}{|c|}{.70} \\
\hline $\mathrm{p}$ & \multicolumn{4}{|c|}{108} & \multicolumn{4}{|c|}{525} & \multicolumn{4}{|c|}{794} \\
\hline & $\mathrm{M}$ & SD & Min & $\operatorname{Max}$ & $\mathrm{M}$ & SD & Min & Max & $\mathrm{M}$ & SD & Min & Max \\
\hline |diff $\mid$ & .0351 & .0205 & .0000 & .0667 & .1687 & .0995 & .0000 & .3500 & .2841 & .1890 & .0000 & .7000 \\
\hline Block $_{\text {avg }}$ & .5566 & .1589 & .2778 & .9611 & .6093 & .1486 & .2778 & 1.0167 & .6659 & .1591 & .2778 & 1.1556 \\
\hline Method & \multicolumn{12}{|c|}{ Block $_{\text {avg }}$} \\
\hline $\mathrm{p}$ & \multicolumn{4}{|c|}{108} & \multicolumn{4}{|c|}{525} & \multicolumn{4}{|c|}{794} \\
\hline \multirow{5}{*}{$\begin{array}{c}\mid \text { diff } \mid \\
\text { Block }_{\text {avg }}\end{array}$} & $\mathrm{M}$ & SD & Min & $\operatorname{Max}$ & $\mathrm{M}$ & SD & Min & Max & $\mathrm{M}$ & SD & Min & Max \\
\hline & .1096 & .0797 & .0000 & .3222 & .2190 & .1480 & .0000 & .6556 & .2942 & .2029 & .0000 & .9056 \\
\hline & .4026 & .0525 & .2778 & .4778 & .5772 & .1116 & .2778 & .7444 & .6622 & .1526 & .2778 & .9389 \\
\hline & \multicolumn{12}{|c|}{ Hit Rate (\%) } \\
\hline & \multicolumn{4}{|c|}{39.81} & \multicolumn{4}{|c|}{81.14} & \multicolumn{4}{|c|}{96.73} \\
\hline
\end{tabular}

Note. $\mid$ diff $\mid=$ absolute mean difference; $\mathrm{p}=$ number of item-pairs selected; $\mathrm{M}=$ mean; $\mathrm{SD}=$ standard deviation; Min = minimum; Max = maximum; Hit Rate $(\%)=$ percentage of item-pairs selected with Block $k_{\text {avg }}$ that were also selected with |diff|. 


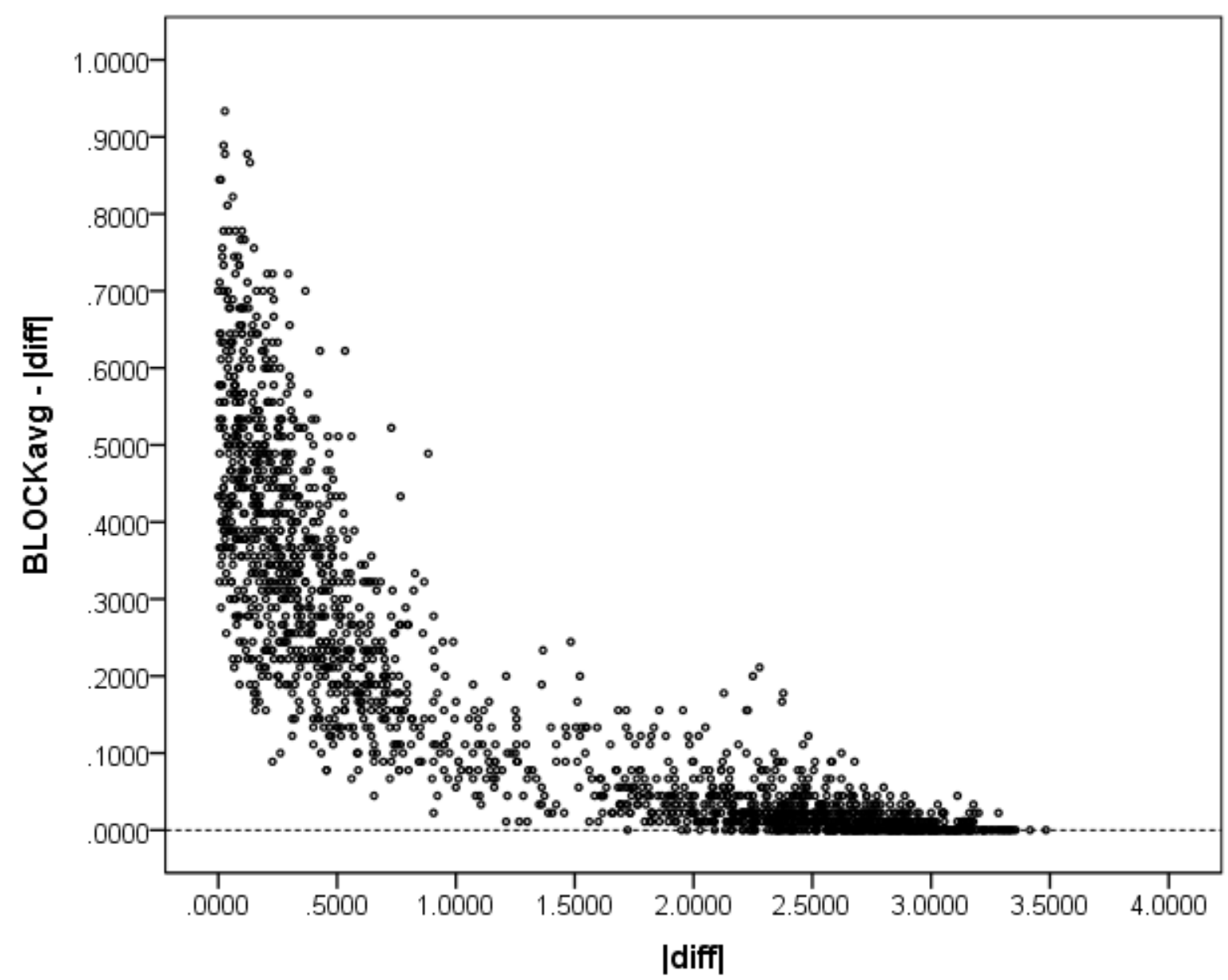

Figure 1. Bivariate scatterplot of the absolute mean difference (|diff $\mid)$ vs. difference between the averaged block distance and the absolute mean difference (Block $\left.\mathrm{avg}_{\text {- }}|\operatorname{diff}|\right)$. Dotted line represents the reference line of equal $\mid$ diff $\mid$ and Block $k_{\text {avg values }}\left(\right.$ Block $\left._{\text {avg }}-|\operatorname{diff}|=0\right)$. 